\title{
Clinical Characteristics of HIV-Associated Optic Neuritis
}

This article was published in the following Dove Press journal:

International Medical Case Reports Journal

\section{Panitha Jindahra' \\ Angsana Phuphuakrat $\mathbb{D}^{2}$ \\ Thanapol Tangjaisanong ${ }^{3}$ \\ Sukanya Siriyotha ${ }^{4}$ \\ Tanyatuth Padungkiatsagul $\mathbb{D}^{5}$ \\ Kavin Vanikieti ${ }^{5}$ \\ Pisit Preechawat ${ }^{5}$ \\ Anuchit Poonyathalang $\mathbb{D}^{5}$ \\ Somnuek Sungkanuparph (1D ${ }^{6}$ \\ Teeratorn Pulkes $\mathbb{D}^{\prime}$ \\ Supoch Tunlayadechanont ${ }^{\prime}$}

'Division of Neurology, Department of Medicine, Faculty of Medicine Ramathibodi Hospital, Mahidol University, Bangkok 10400, Thailand; ${ }^{2}$ Division of Infectious Diseases, Department of Medicine, Faculty of Medicine Ramathibodi Hospital, Mahidol University, Bangkok 10400, Thailand; ${ }^{3}$ Department of Medicine, Bangkhunthian Geriatric Hospital, Bangkok, I0150, Thailand; ${ }^{4}$ Department of Clinical Epidemiology and Biostatistics, Faculty of Medicine Ramathibodi Hospital, Mahidol University, Bangkok 10400, Thailand; ${ }^{5}$ Department of Ophthalmology, Faculty of Medicine Ramathibodi Hospital, Mahidol University, Bangkok 10400, Thailand; ${ }^{6}$ Chakri Naruebodindra Medical Institute, Faculty of Medicine Ramathibodi Hospital, Mahidol University, Samut Prakan 10540, Thailand
Introduction: Acute optic neuritis $(\mathrm{ON})$ has been increasingly reported in patients infected with human immunodeficiency virus (HIV). We aimed to describe clinical characteristics of HIV-infected patients with ON.

Materials and Methods: This observational retrospective study was performed from January 2008 to January 2016 in a university hospital in Bangkok, Thailand. Demographic data and clinical manifestations were retrospectively reviewed.

Results: We included 10 HIV-infected patients and divided them into two groups: infectious and non-infectious ON. There were six patients in the infectious ON group (five males, mean age 33.6 years, median CD4 cell counts during $\mathrm{ON}$ episodes 36.5 cells/ $\mu \mathrm{L}$, high viral loads, median initial visual acuity [VA] 1.7, median VA difference [initial VA - follow-up VA] 0). Four patients developed cryptococcal meningitis and 2 developed neuro-syphilis. They were treated with amphotericin B plus fluconazole and penicillin G sodium, respectively. There were four patients in the non-infectious ON group (two males, mean age 44.3 years, median CD4 cell count during acute ON 157.5 cells $/ \mu \mathrm{L}$, undetectable viral loads, median initial VA 1.3, and median VA difference 1.2). They were given corticosteroid treatments. There were no statistically significant differences between the two groups with respect to age, sex, and initial VA. There were statistically significant differences in median VA difference and median CD4 cell counts between the two groups $(\mathrm{p}=0.02$ and 0.03 , respectively). There was a significant correlation between CD4 counts and duration in three non-infectious ON patients treated with antiretroviral therapy (ART, $\mathrm{p}<0.001$ ).

Discussion: Patients with infectious ON had low initial CD4 counts and high viral loads. Patients with non-infectious ON had increasing CD4 cell counts and undetectable HIV viral load following ART. The findings are highly suggestive of immune reconstitution inflammatory syndrome. The CD4 cell counts and viral loads may help to identify the type of acute $\mathrm{ON}$ in HIV-infected patients and establish proper therapies.

Keywords: optic neuritis, human immunodeficiency virus, acquired immune deficiency syndrome, CD4 cell count, immune reconstitution inflammatory syndrome

\section{Introduction}

Acute optic neuritis $(\mathrm{ON})$ is an inflammatory disorder of the optic nerve. Immunemediated diseases, including multiple sclerosis (MS) and neuromyelitis optica spectrum disorder (NMOSD), are common causes of acute ON. Certain bacterial, viral, and fungal infections can cause ON, particularly in immunocompromised hosts. Visual recovery varies with the diseases and treatments. ${ }^{1-4}$ Acute ON has been increasingly reported in patients infected with the human immunodeficiency virus (HIV), especially those suffering from acquired immune deficiency syndrome (AIDS). ${ }^{5-7}$ An African study showed that a high proportion of $\mathrm{ON}$ in HIV-infected
Correspondence: Panitha Jindahra Division of Neurology, Department of Medicine, Faculty of Medicine Ramathbodi Hospital, Mahidol University, Bangkok, Thailand

Tel/Fax +66 220I 1386

Email panitha.jin@mahidol.ac.th
International Medical Case Reports Journal 2020:13 609-6/6 
patients was caused by opportunistic infections such as cryptococcosis, tuberculosis, cytomegalovirus disease, toxoplasmosis, etc varicella-zoster. ${ }^{7}$ A few pathological studies demonstrated cryptococcal infiltrations in the optic nerve sheath complexes in AIDS patients with ON. ${ }^{8,9}$ Another ON study revealed HIV and cytomegalovirus infiltrations in the optic nerves of a rhesus monkey with AIDS. ${ }^{10}$ The specific treatment of causative agents is essential in these cases. Besides infections, acute $\mathrm{ON}$ in HIV-infected patients can be caused by non-infectious causes or immune-mediated diseases. These patients show no sign of opportunistic infections and have good visual recovery after steroid treatments. ${ }^{11}$ Some developed MS with relapsing-remitting patterns later in the course of their illnesses. ${ }^{12}$ It is essential to clinically differentiate between the infectious and immune-mediated $\mathrm{ON}$ as their treatments significantly differ. CD4 cell counts are the best measurement of a patient's immune status and the risk of opportunistic infections. In HIV stage 1, the immune system is still intact and autoimmune disorders may develop. In stage 2, CD4 cell counts are declining without overt manifestations of AIDS. In stage 3, CD4 cell count less than 200 cells $/ \mu \mathrm{L}$ and the development of AIDS are predictors of opportunistic infections. Autoimmune disorders are not found in this stage. In stage 4, there is evidence of a resurgence of autoimmune disorders following highly active anti-retroviral therapy and immune restoration. ${ }^{13}$ Our goal in this study was to describe clinical characteristics of HIV-infected patients with acute ON.

\section{Materials and Methods}

\section{Participants}

This was an observational retrospective study of HIVinfected patients who developed acute ON. We included patients who received treatments from infectious disease and neuro-ophthalmology clinics, Ramathibodi Hospital, a university hospital in Bangkok, Thailand, from January 2008 to January 2016. Inclusion criteria were a history of acute ON and HIV seropositivity. The patients were divided into two groups, namely, infectious and noninfectious ON. Infectious ON was defined as the presence of HIV seropositivity, clinical presentation of acute $\mathrm{ON}$, radiological evidence of acute $\mathrm{ON}$, or enhancement of the affected optic nerves on contrast-enhanced T1-weighted orbital MRI with fat suppression (CETWFS) and evidence of CNS infection. Non-infectious ON was defined as the presence of HIV seropositivity, clinical presentation of acute
$\mathrm{ON}$, radiological evidence of acute $\mathrm{ON}$, and no evidence of CNS infection. The diagnosis of $\mathrm{ON}$ was made clinically based on symptoms and signs. Patients usually develop subacute visual loss, with/without pain on eye movement, dyschromatopsia, positive relative afferent pupillary defect (in unilateral or asymmetrical ON), visual field defect, with/ without optic disc swelling. We included papillitis optica and retrobulbar $\mathrm{ON}$ as optic neuritis. Magnetic resonance imaging of the orbits confirms acute ON (please see further details in the magnetic resonance imaging part). The diagnosis of central nervous system (CNS) infection was based on history and physical examinations, which were confirmed with MRIs, laboratory investigations, and cerebrospinal fluid (CSF) analysis. In the non-infectious group, there was no evidence of CNS infection in history and physical examinations, MRIs, laboratory investigations, and CSF analysis (normal). Demographic and clinical data including age, sex, antiretroviral therapy (ART), HIV viral loads, CD4 cell counts, bilaterality of $\mathrm{ON}$, initial visual acuity (VA), VA after one year follow-up, visual field tests, and other ophthalmologic examinations were obtained from medical records retrospectively. The initial and follow-up VAs were measured in each eye using a standard Snellen chart, which was converted into a logarithm of the minimum angle of resolution (LogMAR) for statistical analysis. VA difference (initial VA - follow-up VA) was calculated. Visual field tests were performed using either the Humphrey field analyzer (Carl Zeiss Meditec, Inc, Dublin, Calif) or Goldmann perimetry (Octopus perimetry, Haag-Streit Diagnostics, Switzerland).

\section{Magnetic Resonance Imaging}

Magnetic resonance imaging (MRI) of the brain and orbits were reviewed retrospectively. The MRI examinations were performed on two different scanners, a 3.0T scanner (Ingenia; Philips Healthcare, Best, the Netherlands) and a 1.5T scanner (Signa TwinSpeed; GE Healthcare), using our standard brain and orbit MRI protocols that included axial and coronal contrast-enhanced T1-weighted images with fat suppression (CE-T1W/FS), T2-weighted images with fat suppression and axial fluid-attenuation inversion recovery images (FLAIR). Enhancement of the optic nerve on CE-T1W/FS is highly sensitive for acute ON detection.

\section{Blood Tests}

Serum HIV screening tests, CD4 cell counts, rapid plasma reagin (RPR), Treponema pallidum hemagglutination assay (TPHA), aquaporin4-IgG, erythrocyte sedimentation 
rate, complete blood count, liver function test, and renal function test were performed in all cases.

\section{Cerebrospinal Fluid}

CSF analysis was done, including cell counts, cell differentiation, Gram stain, protein, sugar, India ink, aerobic culture, venereal disease research laboratory test (VDRL), TPHA, cryptococcal antigen, antibody to Toxoplasma gondii, polymerase chain reaction to Mycobacterium tuberculosis, mycobacteria culture and fungal culture. CSF culture was the mainstay in the diagnosis of infectious meningitis. The diagnosis of cryptococcal meningitis was based on a positive CSF cryptococcal antigen test and/or a positive India ink test. The neurosyphilis diagnosis was based on CSF white blood cells $\geq 20$ cells/ $\mu \mathrm{L}$, a reactive CSF VDRL, and/or a positive CSF TPHA.

\section{Ethical Issues}

The study was approved by the Ethical Clearance Committee on Human Rights Related to Research Involving Human Subjects, Faculty of Medicine Ramathibodi Hospital, Mahidol University (Trial number MURA2019/156). The patients were informed about the purpose of the study. Consent was obtained from the study participants prior to study commencement.

\section{Statistical Analysis}

Descriptive analyses were performed for demographic data including age, sex, bilaterality of $\mathrm{ON}$, initial VA, followup VA, VA difference (initial VA - follow-up VA), and ophthalmological examinations. Descriptive statistics were presented as mean (SD) and median (range) for continuous variables. Student's $t$-test and Fisher's exact were analyzed to compare age and sex between the two groups. Medians of CD4 counts during acute $\mathrm{ON}$ were calculated in both groups. Mann-Whitney $U$-test was employed to compare the median of CD4 counts during acute ON, initial VA, and VA difference (initial VA - follow-up VA) between the two groups. Multilevel mixed-effects linear regression analysis was analyzed to find a correlation between CD4 counts and duration in the non-infectious ON group. A p-value of less than 0.05 was considered as statistical significance. Stata version 15.5 (College Station, Tx: Statacorp LLC.) was used in the analysis.

\section{Results}

Ten HIV-infected patients with acute ON were included in the study (Table 1, Figures 1 and 2). There were seven males and three females aged 20-51 years. There were six patients in the infectious $\mathrm{ON}$ group (five males, one female, age 20-39 years [mean 33.6, SD 7.4], cases number [NO.] 1-6). Cases NO. 1-6 either received no ART or had poor compliance to ART. They presented with simultaneous acute $\mathrm{ON}$ and CNS infection (four patients with cryptococcal meningitis and two with neuro-syphilis). The median CD4 cell counts during ON episodes was 36.5. HIV viral loads were measured in two patients (400 and 1172 copies $/ \mathrm{mL}$ ). Five patients developed bilateral ON. All affected eyes $(n=11)$ in this group developed swollen discs, and eight developed disc hemorrhages. Of these 11 eyes, generalized depression of the VF was found in nine and peripheral restriction in two. The median initial VA of the affected eyes was 1.7 (range 0.3-1.7). The median follow-up VA of the affected eyes was 1.7 (range 0-1.7). The median VA difference (initial VA - follow-up VA) of the affected eyes was 0 (range -1.4 to 0 ). Anterior uveitis was present in a case with neuro-syphilis but absent in the others. Other ophthalmological findings were unremarkable. Cryptococcal meningitis and neuro-syphilis were treated successfully with amphotericin B plus fluconazole and penicillin $G$ sodium, respectively. An optic nerve sheath fenestration was carried out in an eye of a patient with cryptococcal meningitis. The VA of that eye changed from 2.3 to 1.7 postoperatively.

There were four patients in the non-infectious $\mathrm{ON}$ group (two males, two females, aged 32-51 years [mean 44.3, SD 8.5], cases NO. 7-10). None of them developed an opportunistic infection. There were two patients with isolated $\mathrm{ON}$, one with relapsing isolated $\mathrm{ON}$ and one with seropositive neuromyelitis optica spectrum disorder associated ON (NMOSD-ON). Cases NO. 7-9 received ART [tenofovir disoproxil fumarate (TDF)/3TC/NVP; TDF/ emtricitabine (FTC)/efavirenz (EFV); TDF/FTC/boosted lopinavir (LPV/r)] before developing acute ON. They developed acute ON 12-19 months (median 18) after ART initiation. Following ART initiation, CD4 cell counts gradually increased from the moment of HIV diagnosis to the onset of acute $\mathrm{ON}(15,83,113,89$ cells/ $\mu \mathrm{L}$ in case NO. 7; 40, 107, cells/ $\mu \mathrm{L}$ in case NO. 8 and 42, 77, 89, 107, 208 cells $/ \mu \mathrm{L}$ in case NO. 9). Their CD4 cell counts continued to rise after the ON episodes (207, 200, 208 cells/ $\mu \mathrm{L}$ in cases NO.7-9, respectively). (Figure 3) The initial 


\begin{tabular}{|c|c|c|c|c|c|c|c|c|c|c|c|c|}
\hline 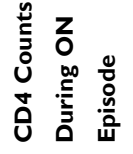 & & F & $\stackrel{\sim}{\sim}$ & సి & $\forall$ & 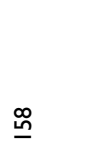 & $\simeq$ & & ळ & 으 & $\stackrel{\infty}{\sim}$ & $\bar{m}$ \\
\hline 总 & & 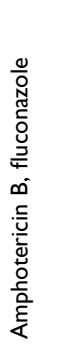 & 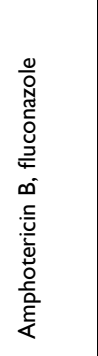 & 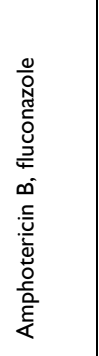 & 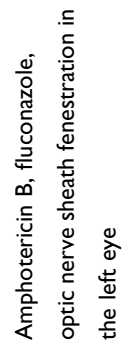 & 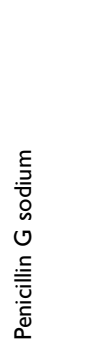 & 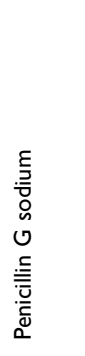 & & 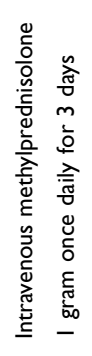 & 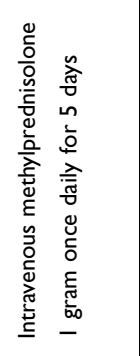 & 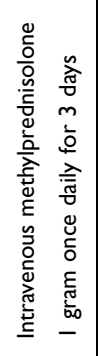 & 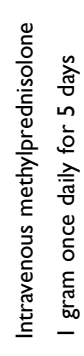 \\
\hline 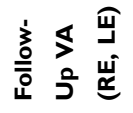 & & $\stackrel{\check{m}}{\bar{m}}$ & 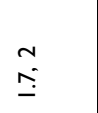 & $\begin{array}{l}\cong \\
\cong\end{array}$ & $\begin{array}{l}= \\
\frac{\infty}{0}\end{array}$ & $\begin{array}{l}0 \\
0\end{array}$ & $\frac{0}{-}$ & & $\begin{array}{l}\stackrel{m}{0} \\
\overline{0}\end{array}$ & $\begin{array}{l}\overline{0} \\
\overline{0}\end{array}$ & $\frac{0}{-1}$ & $\frac{0}{-1}$ \\
\hline 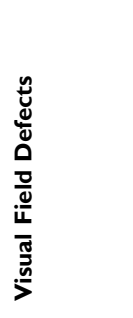 & & 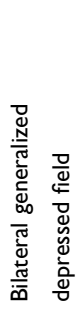 & 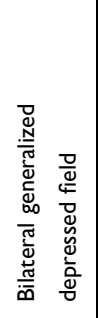 & 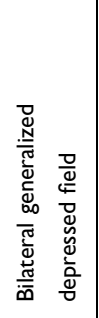 & 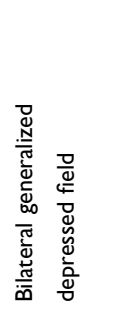 & 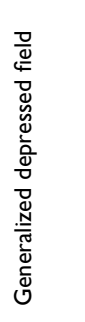 & 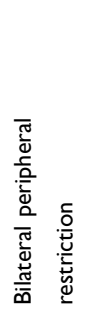 & & 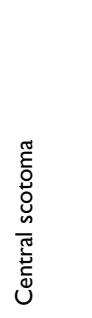 & 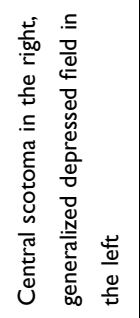 & 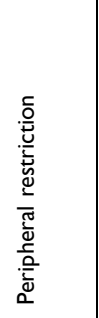 & 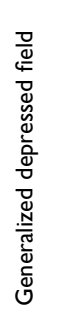 \\
\hline 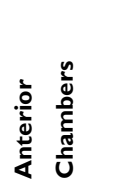 & & 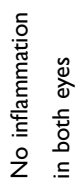 & 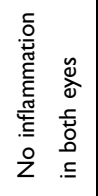 & 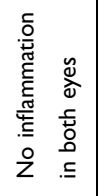 & 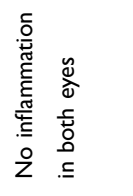 & 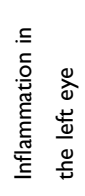 & 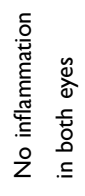 & & 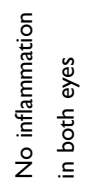 & 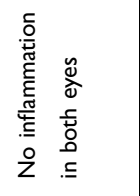 & 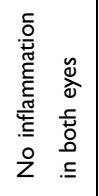 & 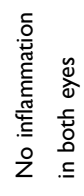 \\
\hline 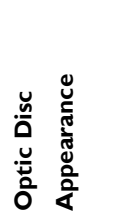 & & 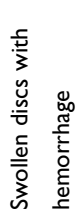 & 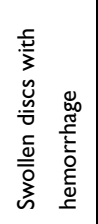 & 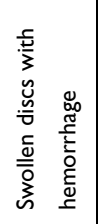 & 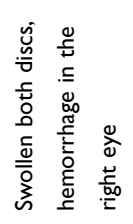 & 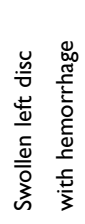 & 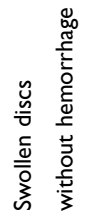 & & 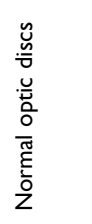 & 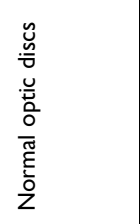 & 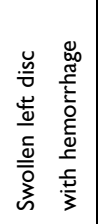 & 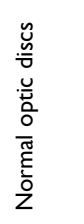 \\
\hline 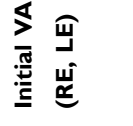 & \multirow{6}{*}{ 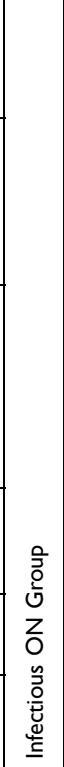 } & $\begin{array}{l}\cong \\
\cong\end{array}$ & $\stackrel{2}{\check{2}}$ & $\begin{array}{l}m \\
0 \\
\infty \\
\infty \\
0 \\
0\end{array}$ & $\begin{array}{l}\stackrel{m}{N} \\
\stackrel{-}{=}\end{array}$ & $\begin{array}{l}\infty \\
\infty \\
0 \\
0 \\
0\end{array}$ & $\begin{array}{l}m \\
\text { m } \\
\text { fं }\end{array}$ & \multirow{6}{*}{ 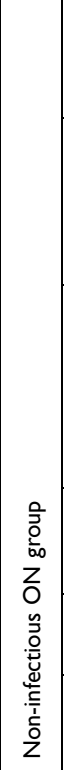 } & $\begin{array}{l}\hat{0} \\
\dot{0}\end{array}$ & $\stackrel{\underline{s}}{\underline{m}}$ & $\begin{array}{l} \pm \\
0 \\
0 \\
0\end{array}$ & 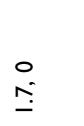 \\
\hline 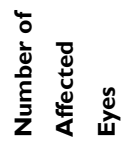 & & $\sim$ & $\sim$ & $\sim$ & $\sim$ & - & N & & - & $N$ & - & - \\
\hline 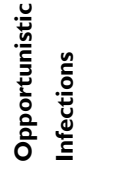 & & 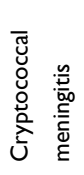 & 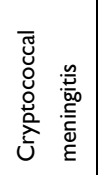 & 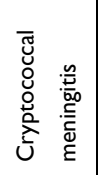 & 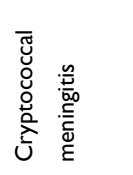 & 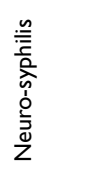 & 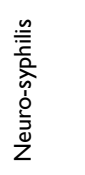 & & 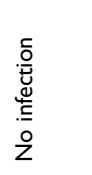 & 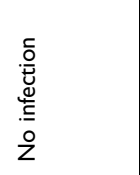 & 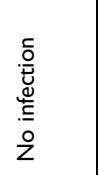 & 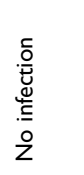 \\
\hline ஸ̊ & & $\frac{\frac{\omega}{\omega}}{\Sigma}$ & $\frac{\frac{\omega}{\pi}}{\sum}$ & $\begin{array}{l}\frac{0}{\tilde{E}} \\
\frac{\tilde{E}}{\sqcup}\end{array}$ & $\frac{\frac{\omega}{\pi}}{\Sigma}$ & $\frac{\frac{\omega}{\omega}}{\Sigma}$ & $\frac{\frac{\omega}{\omega}}{\Sigma}$ & & $\frac{\frac{\omega}{N}}{\Sigma}$ & $\begin{array}{l}\frac{0}{\tilde{E}} \\
\frac{\tilde{E}}{\nu}\end{array}$ & $\frac{\frac{\omega}{\pi}}{\Sigma}$ & $\begin{array}{l}\frac{0}{\widetilde{\varpi}} \\
\frac{\tilde{W}}{L}\end{array}$ \\
\hline 品 & & $\stackrel{\infty}{m}$ & 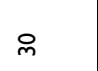 & 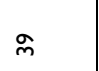 & ని & $\hat{m}$ & $\stackrel{\infty}{m}$ & & $\bar{n}$ & $q$ & ஜ & $\widetilde{m}$ \\
\hline $\begin{array}{l}\stackrel{u}{0 ~} \\
\text { Jू }\end{array}$ & & - & $N$ & $m$ & $\sigma$ & in & 0 & & $n$ & $\infty$ & $\sigma$ & 은 \\
\hline
\end{tabular}




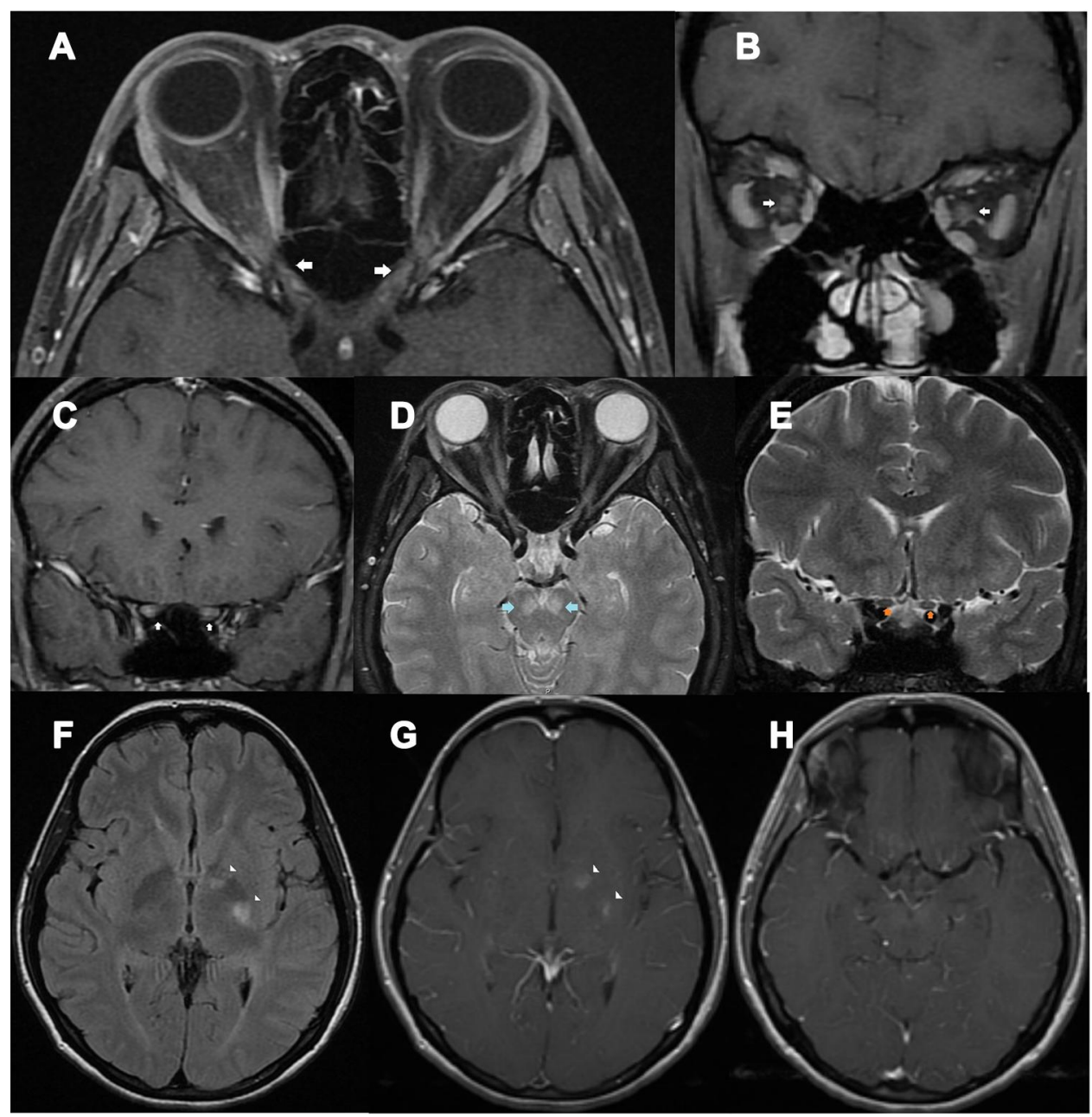

Figure I A 39-year-old HIV-infected female presented with bilateral optic neuritis and cryptococcal meningitis. (A) axial contrast-enhanced TI-weighted images with fat suppression showed enhancement of both optic nerves at the intraorbital, intracanal, and intracranial segments (arrows); (B) coronal contrast-enhanced TI-weighted images with fat suppression showed enhancement of both optic nerves at the intraorbital segments (arrows); (C) coronal contrast-enhanced TI-weighted images with fat suppression showed enhancement of both optic nerves at the intracranial segments (arrows); (D) Axial T2-weighted images with fat suppression showed signal hyperintensity at both cerebral peduncles (arrows) and intraorbital optic nerves; (E) Coronal T2-weighted images with fat suppression showed intracranial optic nerve signal hyperintensity (arrows); (F) axial fluid-attenuation inversion recovery images of the brain showed signal hyperintensity at the genu and posterior limb of left internal capsule (arrowheads); (G) axial contrast-enhanced TI-weighted images of the brain showed abnormal enhancement at the genu and posterior limb of left internal capsule (arrowheads); (H) axial contrast-enhanced TI-weighted images of the brain showed mild leptomeningeal enhancement, suggestive of meningitis.

HIV viral loads were $>500,000$ copies $/ \mu \mathrm{L}$ and became undetectable $(<40$ copies $/ \mathrm{mL})$ during acute ON. Their serum aquaporin 4-IgG antibodies and other serum autoimmune antibodies were negative. Case NO. 10 was newly diagnosed with asymptomatic HIV infection and did not receive ART. The patient's CD4 cell counts were 371 cells $/ \mu \mathrm{L}$ during acute $\mathrm{ON}$ and were aquaporin4-IgG seropositive. The patient was diagnosed with NMOSD-ON. The median CD4 cell count during acute ON was 157.5 cells $/ \mu \mathrm{L}$. A patient in this group developed bilateral $\mathrm{ON}$. Of all five affected eyes, optic discs appeared normal in four; swelling and hemorrhage occurred in one. Generalized depression of the VF was found in two eyes, central scotoma in two and peripheral restriction in one The median initial VA of the affected eyes was 1.3 (range 0.4-1.7). The median follow-up VA of the affected eyes was 0.1 (range $0-0.3$ ). The median VA difference (initial VA - follow-up VA) of the affected eyes was $1.2(0.4-0.6)$. Other ophthalmological findings were unremarkable. Intravenous methylprednisolone was given in all patients for 3-5 days, followed by a short course of oral prednisolone.

There was no statistically significant difference of the mean age between the two groups $(\mathrm{p}=0.07)$. There was no statistically significant difference of sex between the two groups $(\mathrm{p}=0.2)$. There was a statistically significant 


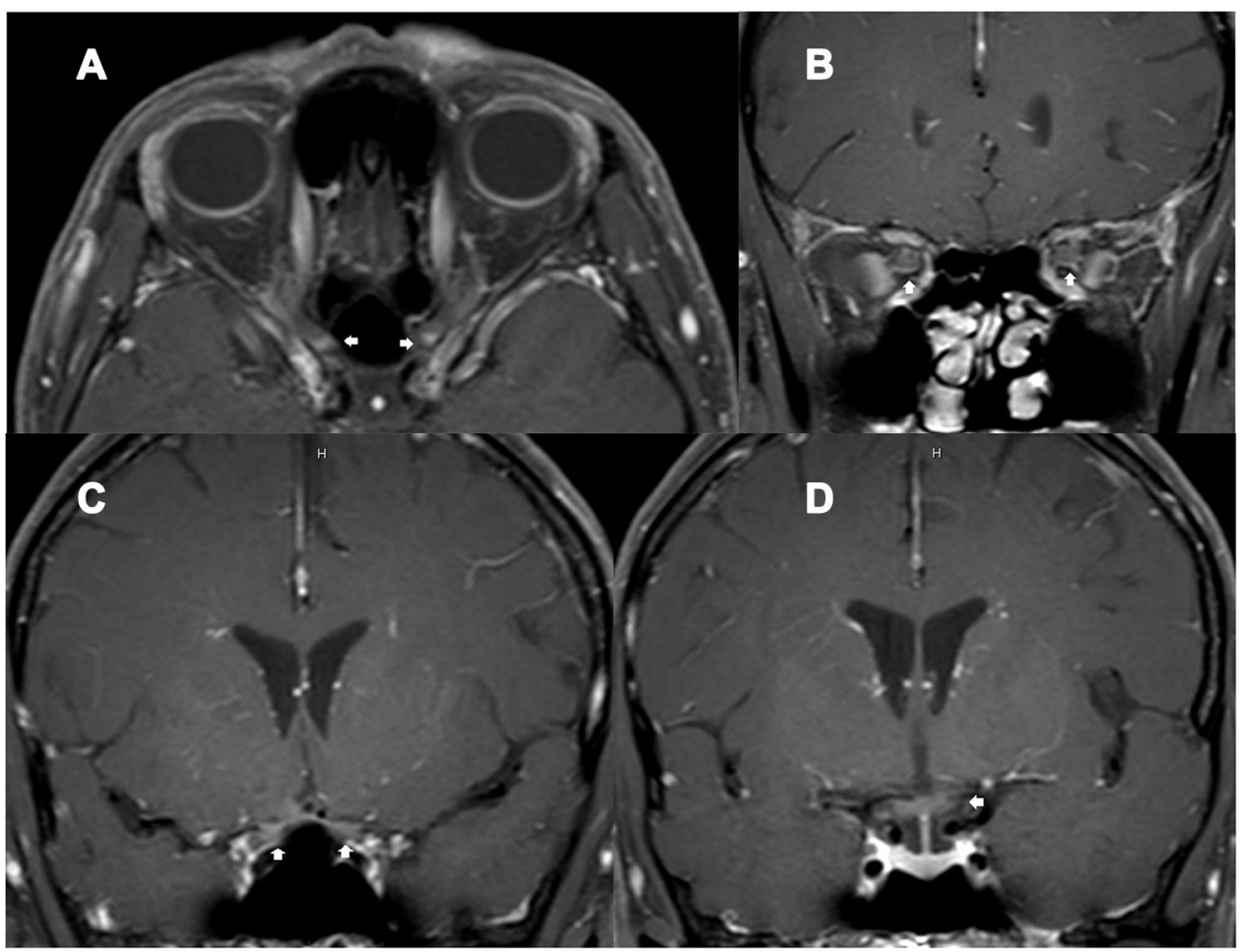

Figure 2 A 49-year-old HIV-infected female, who received antiretroviral therapy with well-controlled HIV viral load, presented with bilateral optic neuritis (immune reconstitution inflammatory syndrome-associated optic neuritis). (A) axial contrast-enhanced TI-weighted images with fat suppression showed enhancement of both optic nerves at the intracanal and intracranial segments (arrows); (B) coronal contrast-enhanced TI-weighted images with fat suppression showed enhancement of both optic nerves at the intraorbital segments (arrows); (C) coronal contrast-enhanced TI-weighted images with fat suppression showed enhancement of both optic nerves at the intracranial segments (arrows); (D) coronal contrast-enhanced TI-weighted images with fat suppression showed enhancement of the chiasm (arrow).

\section{Non-infectious Optic Neuritis Group}

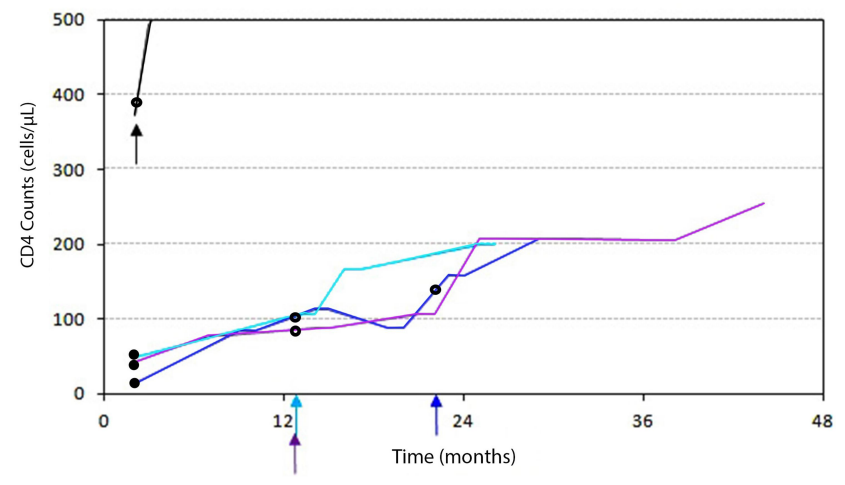

Figure 3 Diagram showing individual trajectories of the CD4 cell count over time in the non-infectious (immune-mediated) ON group. Each color line and the corresponding arrow color represents each patient. Small arrows represent acute ON attacks. Black dots represent antiretroviral initiation. Black rings represent steroid treatments. Three patients (blue, light blue, and purple lines) successfully achieved HIV infection control. They developed acute ON while their CD4 cell counts were rising, and HIV viral load became undetectable. A female patient (black line), who did not receive ART, developed NMOSD-ON while her CD4 cell counts were $37 \mathrm{I}$ cells/ $\mu \mathrm{L}$. All the patients in this group did not have opportunistic infections and were responsive to corticosteroid treatments.

difference of the median CD4 counts during acute ON between the infectious and non-infectious ON groups $(\mathrm{p}=0.03)$. Multilevel mixed-effects linear regression analysis revealed a significant correlation between CD4 counts and duration (months) in the three non-infectious ON patients with ART $(\mathrm{p}<0.001)$. There was no statistically significant difference of the median initial VA in the affected eyes between the two groups $(\mathrm{p}=0.8)$. There was a statistically significant difference of the median VA difference (initial VA - follow-up VA) in the affected eyes between the two groups $(\mathrm{p}=0.02)$.

\section{Discussion}

There is increasing evidence of HIV-associated ON, particularly in African and Asia Pacific countries. Among all etiologies of ON, the frequency of HIV-associated ON was around $25 \%$ in an African study but 3.9\% among adult subjects in our institute. ${ }^{7}$ This study highlighted the importance of HIV stages and immune status in different causes of acute ON. Here we showed that patients in the infectious ON group developed opportunistic infections as a result of damage to their immune systems from advanced HIV infection (AIDS). Their initial CD4 cell counts were low with high HIV viral loads. On the contrary, there was an increasing trend of CD4 counts after ART initiation in three patients with non-infectious ON (from before ON attack to several months after steroid treatments). Their 
HIV viral loads were undetectable. Furthermore, another patient in the non-infectious ON group, who had relatively high CD4 cell counts during ON attack, developed NMOSD-ON. Their vision was well restored following steroid treatments. These findings suggested that the immune processes play a key role in the pathophysiology of the non-infectious $\mathrm{ON}$.

Infectious ON coincided with CNS infection, such as cryptococcal meningitis and neuro-syphilis. The organisms invade the optic nerve sheath complexes, leading to permanent nerve damage and poor visual outcome. However, raised intracranial pressure in cryptococcal meningitis can also compromise the optic nerve to a certain degree. Noted that the VA in the eye improved following optic nerve fenestration. The infectious $\mathrm{ON}$ patients developed more optic disc swelling with hemorrhages and poorer visual outcomes than the non-infectious ON patients. The findings of this research were consistent with previous studies. ${ }^{7,8}$

Possible mechanisms of the non-infectious $\mathrm{ON}$ are immune reconstitution inflammatory syndrome (IRIS) and autoimmune ON. The term IRIS describes a phenomenon of clinical deterioration following successful viral suppression and recovered immune status in HIVinfected patients receiving ART. IRIS approximately occurs in $20-35 \%$ of HIV-infected patients treated with ART, of which $1 \%$ develop CNS-IRIS. ${ }^{14}$ CNS-IRIS is frequently associated with opportunistic infections such as infections due to Cryptococcus neoformans, Mycobacterium tuberculosis, and JC virus. CNS-IRIS can also occur in the absence of opportunistic infections such as encephalitis, demyelination, MS, deafness, dementia, and leukoencephalopathy. ${ }^{15}$ However, IRISassociated $\mathrm{ON}$ is not well recognized and has been recently reported only in one case. ${ }^{16}$ After ART initiation, the HIV viral load of this reported case had significantly dropped from 569,000 copies/mL to 891 copies/mL during ON episodes. CD4 cell counts had slightly increased from 7 to 32 cells $/ \mu \mathrm{L}$. Despite the low CD4 cell counts, it is possible that a small subset had been released, leading to rebound inflammation. ${ }^{16}$ The mechanisms of CNS-IRIS are not well defined. Pathological studies show predominant CD8+ lymphocytic infiltrates, astrocytosis, and neuronal loss in CNS-IRIS lesions. ${ }^{15}$ It is likely that viruses enter into the brain or optic nerve during immune depletion. The immune restoration may target residual viruses or HIV-Tat protein in the CNS/optic nerve. ${ }^{14}$ Corticosteroids are the treatment of choice in this condition.

\section{Conclusions}

Patients with infectious ON had low initial CD4 counts and high viral loads. Patients with non-infectious $\mathrm{ON}$ had increasing CD4 cell counts and undetectable HIV viral load following ART. The findings are highly suggestive of immune reconstitution inflammatory syndrome. The CD4 cell counts and viral loads may help identify the type of acute $\mathrm{ON}$ in $\mathrm{HIV}$-infected patients and establish the proper therapy.

\section{Acknowledgments}

We thank Jeffrey $\mathrm{K}$ from Enago for editing a draft of this manuscript. This study was supported by Division of Neurology, Department of Medicine, Faculty of Medicine Ramathbodi Hospital, Mahidol University.

\section{Disclosure}

The authors report no conflicts of interest in this work.

\section{References}

1. Beck RW, Gal RL, Bhatti MT, et al. Visual function more than ten years after optic neuritis: experience of the optic neuritis treatment trial. Am J Ophthalmol. 2004;137(1):77-83.

2. Pau D, Zubidi N, Yalamanchili S, Plant GT, Lee AG. Optic neuritis. Eye. 2011;25(7):833-842. doi:10.1038/eye.2011.81

3. Petzold A, Wattjes MP, Costello F, et al. The investigation of acute optic neuritis: a review and proposed protocol. Nat Rev Neurol. 2014;10(8):447-458. doi:10.1038/nrneurol.2014.108

4. Gaier ED, Boudreault K, Rizzo JF, Falardeau J, Cestari DM. Atypical optic neuritis. Curr Neurol Neurosci Rep. 2015;15(12):76.

5. Moodley A, Rae W, Bhigjee A. Visual loss in HIV-associated cryptococcal meningitis: a case series and review of the mechanism involved. South Afr J HIV Med. 2015;16(1):305. doi:10.4102/sajhivmed.v16i1.305

6. Duda J, Castro J. Bilateral retrobulbar optic neuritis caused by varicella zoster virus in a patient with AIDS. Br J Med Med Res. 2015;5 (11):1381-1386. doi:10.9734/BJMMR/2015/14259

7. Mustak H, Cook C. Clinical profile and outcomes of optic neuritis in an HIV prevalent urban community in South Africa. Middle East Afr J. 2017;24(3):131-135. doi:10.4103/meajo.MEAJO_133_17

8. Cohen DB, Glasgow BJ. Bilateral optic nerve cryptococcosis in sudden blindness in patients with acquired immune deficiency syndrome. Ophthalmology. 1993;100(11):1689-1694. doi:10.1016/ S0161-6420(93)31416-8

9. Corti M, Solari R, Cangelosi D, et al. Sudden blindness due to bilateral optic neuropathy associated with cryptococcal meningitis in an AIDS patient. Rev Iberoam Micol. 2010;27(4):207-209.

10. García A, Siddappa NB, Li Q, et al. AIDS and optic neuritis in a rhesus monkey infected with the R5 clade C SHIV-1157ipd3N4. J Med Primatol. 2010;39(5):356-360.

11. Burton BJ, Leff AP, Plant GT. Steroid-responsive HIV optic neuropathy. J Neuroophthalmol. 1998;18(1):25-29.

12. Chin JH. Multiple sclerosis and HIV-1 infection. J Neurovirol. 2015;21(4):464-467. doi:10.1007/s13365-015-0335-1

13. Zandman-Goddard G, Shoenfeld Y. HIV and autoimmunity. Autoimmun Rev. 2002;1(6):329-337. doi:10.1016/s1568-9972(02) 00086-1 
14. Johnson $\mathrm{T}$, Nath A. Immune reconstitution inflammatory syndrome and the central nervous system. Curr Opin Neurol. 2011;24 (3):284-290. doi:10.1097/WCO.0b013e328346be57

15. Gray F, Lescure FX, Adle-Biassette H, et al. Encephalitis with infiltration by $\mathrm{CD} 8+$ lymphocytes in HIV patients receiving combination antiretroviral treatment. Brain Pathol. 2013;23(5):525-533. doi:10.1111/bpa.12038
16. Sluch IM, LaCroix J, Patel AD. Bilateral optic neuritis: a new twist on immune reconstitution inflammatory syndrome. Can J Ophthalmol. 2017;52(3):e104-e107.

\section{Publish your work in this journal}

The International Medical Case Reports Journal is an international, peer-reviewed open-access journal publishing original case reports from all medical specialties. Previously unpublished medical posters are also accepted relating to any area of clinical or preclinical science. Submissions should not normally exceed 2,000 words or 4 published pages including figures, diagrams and references. The manuscript management system is completely online and includes a very quick and fair peer-review system, which is all easy to use. Visit http://www.dovepress.com/testimonials.php to read real quotes from published authors. 